\title{
2M1155-79 (= T CHAMAELEONTIS B): A LOW-MASS, WIDE-SEPARATION COMPANION TO THE NEARBY, “OLD” T TAURI STAR T CHAMAELEONTIS
}

\author{
Joel H. Kastner ${ }^{1}$, Emily A. Thompson ${ }^{1,2}$, Rodolfo Montez JR. ${ }^{1}$, Simon J. Murphy ${ }^{3}$, Michael S. Bessell ${ }^{3}$, \\ AND GIUSEPPE GERMANO SACCO ${ }^{1,4}$ \\ ${ }^{1}$ Center for Imaging Science and Laboratory for Multiwavelength Astrophysics, Rochester Institute of Technology, \\ 54 Lomb Memorial Drive, Rochester, NY 14623, USA; jhk@ cis.rit.edu \\ ${ }^{2}$ West Irondequoit High School, Rochester, NY 14617, USA \\ ${ }^{3}$ RSAA, Mount Stromlo Observatory, The Australian National University, ACT 2611, Australia \\ ${ }^{4}$ INAF-Osservatorio Astrofisico di Arcetri, Largo E. Fermi 5, Firenze 50125, Italy \\ Received 2011 December 19; accepted 2012 February 2; published 2012 February 21
}

\begin{abstract}
The early-K star T Cha, a member of the nearby $(D \approx 100 \mathrm{pc}) \epsilon$ Cha Association, is a relatively "old" (age $\sim 7 \mathrm{Myr}$ ) $\mathrm{T}$ Tauri star that is still sporadically accreting from an orbiting disk whose inner regions are now evidently being cleared by a close, substellar companion. We report the identification, via analysis of proper motions, serendipitous $\mathrm{X}$-ray imaging spectroscopy, and follow-up optical spectroscopy, of a new member of the $\epsilon$ Cha Association that is very likely a low-mass companion to $\mathrm{T}$ Cha at a projected separation of $\sim 38 \mathrm{kAU}$. The combined X-ray and optical spectroscopy data indicate that the companion, T Cha B (=2M1155-79), is a weak-lined T Tauri star (wTTS) of spectral type M3 and age $\lesssim 10$ Myr. The serendipitous X-ray (XMM-Newton) observation of T Cha B, which targeted T Cha, also yields serendipitous detections of two background wTTS in the Chamaeleon cloud complex, including one newly discovered, low-mass member of the Cha cloud pre-main-sequence (pre-MS) population. T Cha becomes the third prominent example of a nearby, "old" yet still actively accreting, K-type pre-MS star/disk system (the others being TW Hya and V4046 Sgr) to feature a low-mass companion at very large (12-40 kAU) separation, suggesting that such wide-separation companions may affect the conditions and timescales for planet formation around solar-mass stars.
\end{abstract}

Key words: binaries: visual - circumstellar matter - protoplanetary disks - stars: pre-main sequence

Online-only material: color figures

\section{INTRODUCTION}

The majority of stars likely form in binary systems (e.g., Mathieu 1994; Tohline 2002). Given the recent, rapid improvement in our knowledge of the demographics of nearby ( $D \lesssim 100 \mathrm{pc}$ ), young (age $\sim 10-30 \mathrm{Myr}$ ) stellar groups (e.g., Torres et al. 2008; Zuckerman et al. 2011, and references therein), we now have the opportunity to study, at close range, the composition and, perhaps, dissolution of such pre-mainsequence (pre-MS) binaries during or just after the epoch of planet formation. Indeed, initial studies of nearby, young binaries make apparent that binary star interactions could have profound consequences on planet-forming circumstellar environments (e.g., Prato et al. 2001; Andrews et al. 2010; Kastner et al. 2011).

The $\epsilon$ Cha Association member T Cha (spectral type K0, $D=109 \mathrm{pc}$, age $\sim 7 \mathrm{Myr}$; Torres et al. 2008) is a rare example of a nearby pre-MS star that displays evidence for ongoing accretion (other prominent examples are TW Hya, V4046 Sgr, and MP Mus; Torres et al. 2008). The accretion rate of $\mathrm{T}$ Cha is evidently highly variable (and is often undetectably small; Torres et al. 2008; Schisano et al. 2009). Like TW Hya and V4046 Sgr, T Cha is a prototypical example of a "transition disk" object, i.e., a low-mass, pre-MS star orbited by a relatively massive, dusty disk with a large (AU to tens of AU scale) inner hole or gap. Such disk structure is possibly indicative of the presence of young, massive planets (e.g., Dodson-Robinson \& Salyk 2011). Indeed, Huélamo et al. (2011) recently obtained direct evidence (via aperture-masked adaptive optics imaging) for a substellar object orbiting within the inner gap of the T Cha disk.
Following the identification of apparent companions to HR 4796 and V4046 Sgr at projected separations of $\sim 13.5$ and $\sim 12.4$ kAU, respectively (Kastner et al. 2008, 2011), we are searching for distant companions associated with other pre-MS stars within $\sim 100 \mathrm{pc}$ of Earth, particularly nearby pre-MS stars that are still orbited by and accreting from gaseous, circumstellar disks. In this Letter, we report the identification, via analysis of cataloged proper motions (PMs), serendipitous X-ray imaging spectroscopy, and follow-up optical spectroscopy, of a new, M-type member of the $\epsilon$ Cha Association that is most likely a distant (projected separation $\sim 38 \mathrm{kAU}$ ) companion to T Cha.

\section{DATA AND RESULTS}

\subsection{Proper Motions of Red 2MASS Stars Near T Cha}

To search for candidate comoving, wide-separation companions to $\mathrm{T}$ Cha, we made use of the Vizie $\mathrm{R}^{5}$ table browsing and source matching utilities available in TOPCAT. ${ }^{6}$ We first compiled a list of all $2 \mathrm{MASS}^{7}$ sources within $20^{\prime}$ of T Cha that have $K$ magnitudes and $J-K$ colors within the range expected for lowmass (late $\mathrm{K}$ or M-type) stars within $\sim 100 \mathrm{pc}$, i.e., $K \leqslant 12$ and $0.8 \leqslant J-K \leqslant 1.2$. We then used the UCAC3 (Zacharias et al. 2010) PM catalog to select the subset of these 2MASS sources

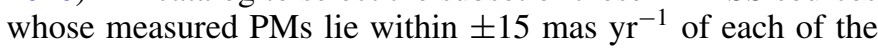

\footnotetext{
5 http://vizier.u-strasbg.fr/viz-bin/VizieR

6 Tool for OPerations on Catalogues And Tables; http://www.star.bris.ac.uk/mbt/topcat/

7 The Two Micron All Sky Survey is a joint project of the University of Massachusetts and the Infrared Processing and Analysis Center/California Institute of Technology and is funded by NASA and the National Science Foundation.
} 
Table 1

Data and Results for T Cha Field XMM Sources ${ }^{\mathrm{a}}$

\begin{tabular}{|c|c|c|c|c|}
\hline & T Cha & $2 \mathrm{M} 1155-79$ & RXJ1158.5-7913 & 2M1158-79 \\
\hline$\mu_{\alpha}\left(\operatorname{mas~yr}^{-1}\right)$ & $-41.2(3.2)$ & $-40.6(5.1)$ & $-18.3(3.8)$ & $-10.1(2.9)$ \\
\hline$\mu_{\delta}\left({\left.\operatorname{mas~} \mathrm{yr}^{-1}\right)}^{\prime}\right.$ & $-6.1(1.4)$ & $-4.7(5.6)$ & $-3.1(1.9)$ & $0.7(2.4)$ \\
\hline$J(\mathrm{mag}, 1.25 \mu \mathrm{m})$ & $8.955(0.027)$ & $11.216(0.022)$ & $9.745(0.024)$ & $12.232(0.026)$ \\
\hline$H(\mathrm{mag}, 1.65 \mu \mathrm{m})$ & $7.860(0.046)$ & $10.465(0.026)$ & $8.959(0.024)$ & $11.544(0.026)$ \\
\hline$K(\mathrm{mag}, 2.2 \mu \mathrm{m})$ & $6.954(0.018)$ & $10.079(0.021)$ & $8.653(0.019)$ & $11.291(0.024)$ \\
\hline$W 1(\mathrm{mag}, 3.4 \mu \mathrm{m})$ & $5.899(0.047)$ & $9.894(0.024)$ & $8.497(0.024)$ & $11.144(0.025)$ \\
\hline$W 2(\mathrm{mag}, 4.6 \mu \mathrm{m})$ & $4.974(0.030)$ & $9.667(0.021)$ & $8.384(0.021)$ & $11.153(0.022)$ \\
\hline$W 3(\mathrm{mag}, 12 \mu \mathrm{m})$ & $4.631(0.017)$ & $9.273(0.033)$ & $8.188(0.024)$ & $10.974(0.092)$ \\
\hline$W 4(\mathrm{mag}, 22 \mu \mathrm{m})$ & $2.601(0.017)$ & $7.195(0.081)$ & $7.864(0.132)$ & $8.683(0.239)$ \\
\hline $\mathrm{EW}, \mathrm{H} \alpha(\AA)$ & $-10.5(0.4)$ & $-6.5(0.5)$ & $-3.1^{\mathrm{b}}$ & $\ldots$ \\
\hline EW, Li $\lambda 6708(\mathrm{~mA})$ & $350(50)$ & $550(100)$ & $600^{\mathrm{b}}$ & $\ldots$ \\
\hline EPIC counts ${ }^{c}$ & $432,660,687$ & $48,148,122$ & $363,441,513$ & $150,276,303$ \\
\hline Count rate ${ }^{c}$ (counts ks ${ }^{-1}$ ) & $132,63,61$ & $13,13,9.3$ & $110,41,45$ & $43,24,25$ \\
\hline$N_{\mathrm{H}}\left(\times 10^{21} \mathrm{~cm}^{-2}\right)$ & $9.7^{\mathrm{d}}$ & $0.16(0-0.79)$ & $0.47(0.30-0.68)$ & $1.5(0.6-2.4)$ \\
\hline$k T_{X}(\mathrm{keV})$ & $\ldots$ & $2.1(1.7-2.8)$ & $0.65(0.60-0.74), 2.3(2.1-2.7)$ & $4.7(3.4-7.6)$ \\
\hline 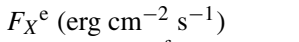 & $2.0 \times 10^{-12 \mathrm{~d}}$ & $1.2 \times 10^{-13}$ & $5.9 \times 10^{-13}$ & $5.9 \times 10^{-13}$ \\
\hline $\log \left(L_{X} / L_{\text {bol }}\right)^{f}$ & $-3.91^{\mathrm{d}}$ & -3.19 & -3.11 & $-2.1:$ \\
\hline
\end{tabular}

Notes.

a Obtained via the observations and analysis described in the text (Sections 2 and 3) unless otherwise indicated.

b EW data from Alcala et al. (1995).

${ }^{c}$ pn, MOS1, and MOS2 total counts and net (background-subtracted) count rates.

${ }^{\mathrm{d}}$ Based on X-ray spectral fit results listed in Güdel et al. (2010).

e Intrinsic ("unabsorbed") X-ray flux.

${ }^{\mathrm{f}}$ Bolometric fluxes for RXJ1158.5 and 2M1158-79 were estimated as described in Section 3.1 (for 2M1155-79). Both the bolometric flux and steady-state X-ray flux of 2M1158-79 are uncertain.

PM components of T Cha. This resulted in a list of six comoving companion candidates to T Cha. Of these candidates, the closest PM match to T Cha is 2MASS 11550485-7919108 (hereafter 2M1155-79); its UCAC3 PM is indistinguishable from that of $\mathrm{T}$ Cha, given the respective measurement uncertainties (Table 1). With an angular separation of 6!36, this candidate is also the closest in proximity to $\mathrm{T}$ Cha. The other five objects with PMs similar to T Cha (and 2M1155-79) all have RA

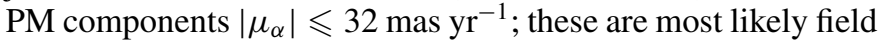
stars unrelated to either the $\epsilon$ Cha Association or the background Chamaeleon cloud T Tauri star population (given that three of the five are undetected in X-rays; see Section 2.2 and Terranegra et al. 1999).

\subsection{XMM-Newton Archival Data}

Low-mass pre-MS stars are luminous X-ray sources, with typical X-ray luminosities (relative to bolometric) in the range $L_{X} / L_{\text {bol }} \sim 10^{-4}-10^{-3}$ (e.g., Kastner et al. 1997). Hence, to confirm that 2M1155-79 is young, we examined an XMM-Newton observation, obtained on UT 2009 March 16, that targeted (and detected) T Cha (Güdel et al. 2010). The observation duration was $11.5 \mathrm{ks}$; the effective exposure times with the European Photon Imaging Camera (EPIC) pn, MOS1, and MOS2 detectors were $3.3,10.6$, and $10.2 \mathrm{ks}$, respectively (the useful pn exposure time being limited due to intervals of high background). The merged $X M M$ /EPIC $0.5-2.0 \mathrm{keV}$ image is displayed in Figure 1, overlaid with the positions and PM vectors of T Cha and the half-dozen bright, red 2MASS stars that have similar UCAC3 PMs (selected as described in Section 2.1). Of these seven stars with similar PMs, only T Cha and 2M1155-79 are detected as X-ray sources (note, however, that two of these PM-selected stars do not fall within the $X M M$ field of view).

The two other luminous X-ray sources in the field, which have UCAC3 catalog PMs that are less than half those of T Cha and 2M1155-79, correspond to 2MASS 11583429-7913175 (= RXJ 1158.5-7913; hereafter RXJ1158.5) and 2MASS 11581646-7931082 (hereafter 2M1158-79). The former is a weak-lined T Tauri star (wTTS) of spectral type K3, previously identified by Alcala et al. (1995) as being associated with the Chamaeleon cloud complex. The Cha clouds and their associated TTS population lie $\sim 160 \mathrm{pc}$ from Earth (Whittet et al. 1997)-i.e., $\sim 60$ pc behind the $\epsilon$ Cha Association-consistent with the small PM of RXJ1158.5 relative to $\epsilon$ Cha member T Cha (Terranegra et al. 1999). Meanwhile, 2M1158-79 has no previous references listed in SIMBAD. ${ }^{8}$ Given its $J H K$ magnitudes and colors relative to those of RXJ1158.5 (Table 1), and the similarity of their PMs, 2M1158-79 is therefore most likely a previously unidentified, lower-mass (early M) member of the Cha cloud wTTS population. PMs are unavailable for potential optical counterparts to the other, weaker X-ray sources in Figure 1.

We used the $X M M$ Scientific Analysis System $\left(\mathrm{SAS}^{9}\right.$ version 10.0.0) to extract pn, MOS1, and MOS2 CCD spectra and responses for the three bright sources that surround $\mathrm{T}$ Cha in the EPIC image (spectral fitting results for $\mathrm{T}$ Cha itself were presented in Güdel et al. 2010). Calibrations were performed using the current calibration files from release note 271, 2010 December 21. Background-subtracted total counts and count rates for each source, as obtained from the pn, MOS1, and MOS2 spectral extractions, are listed in Table 1 (the anomalously low pn count rate of $2 \mathrm{M} 1155-79$ is the result of its position on a bad row of that detector). We summarize the results of simultaneous fits of an absorbed single-component thermal plasma emission model to the three (pn, MOS1, MOS2) EPIC spectra of 2M1155-79 and 2M1158-79 and an absorbed

\footnotetext{
8 The SIMBAD database (http://simbad.u-strasbg.fr/simbad/) is operated at CDS, Strasbourg, France.

9 http://xmm.esa.int/sas
} 


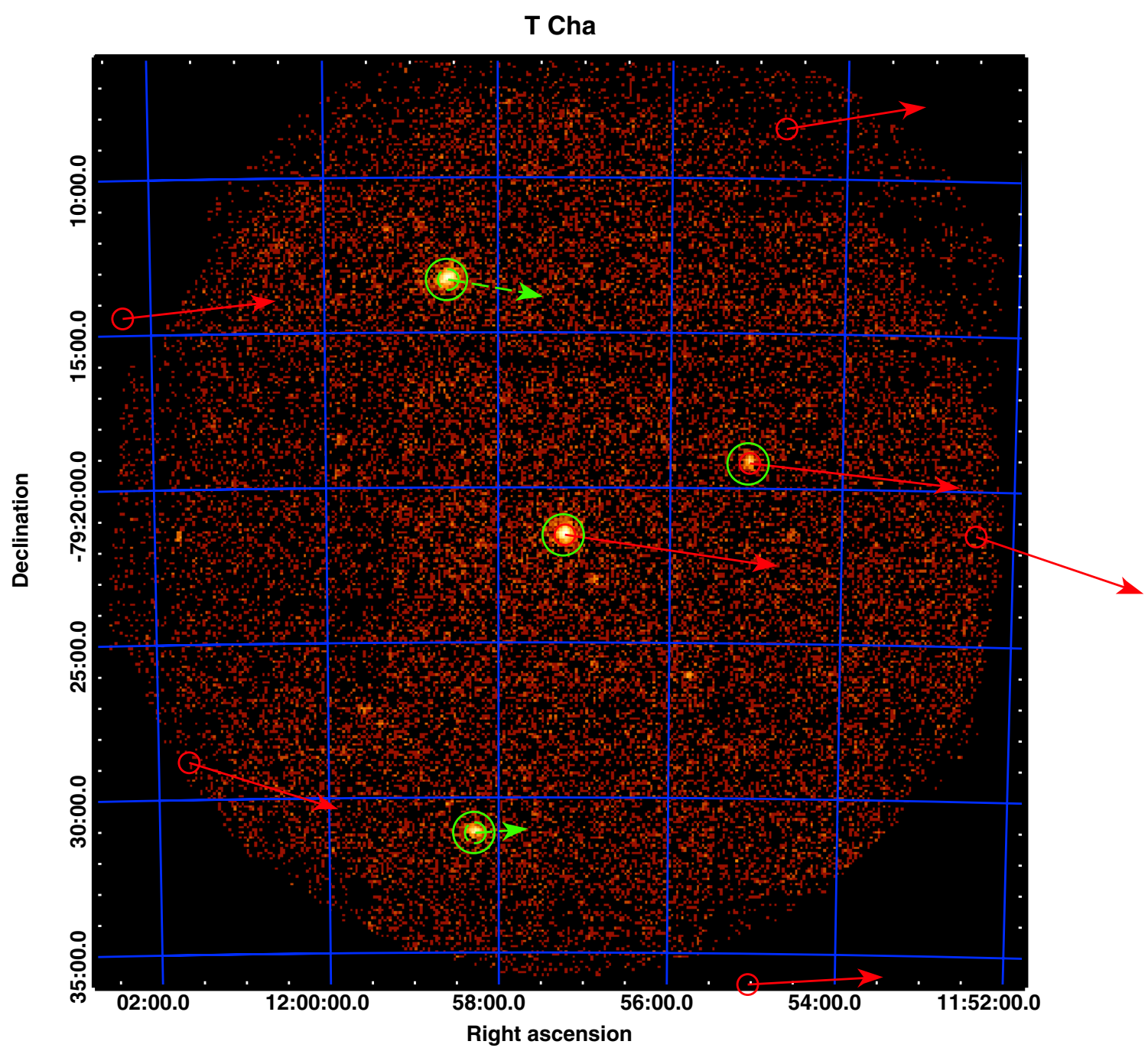

Figure 1. $X M M$ /EPIC $(0.2-12 \mathrm{keV}) \mathrm{X}$-ray image centered on $\mathrm{T}$ Cha; $\mathrm{N}$ is up and $\mathrm{E}$ is to the left, and the imaged field is approximately $18^{\prime}$ in radius. Green circles indicate the four brightest X-ray sources in the field: T Cha (center) and (clockwise from upper left) RXJ1158.5, 2M1155-79, and 2M1158-79. Positions and UCAC3 proper motions (PMs) of field stars with PMs similar to that of T Cha (see Section 2.1) are marked with red circles and vectors, respectively, where vectors represent angular displacements over a time of $10^{4} \mathrm{yr}$. The PMs of the background wTTS (RXJ1158.5 and 2M1158-79; see Section 2.2) are indicated as dashed green vectors. (A color version of this figure is available in the online journal.)
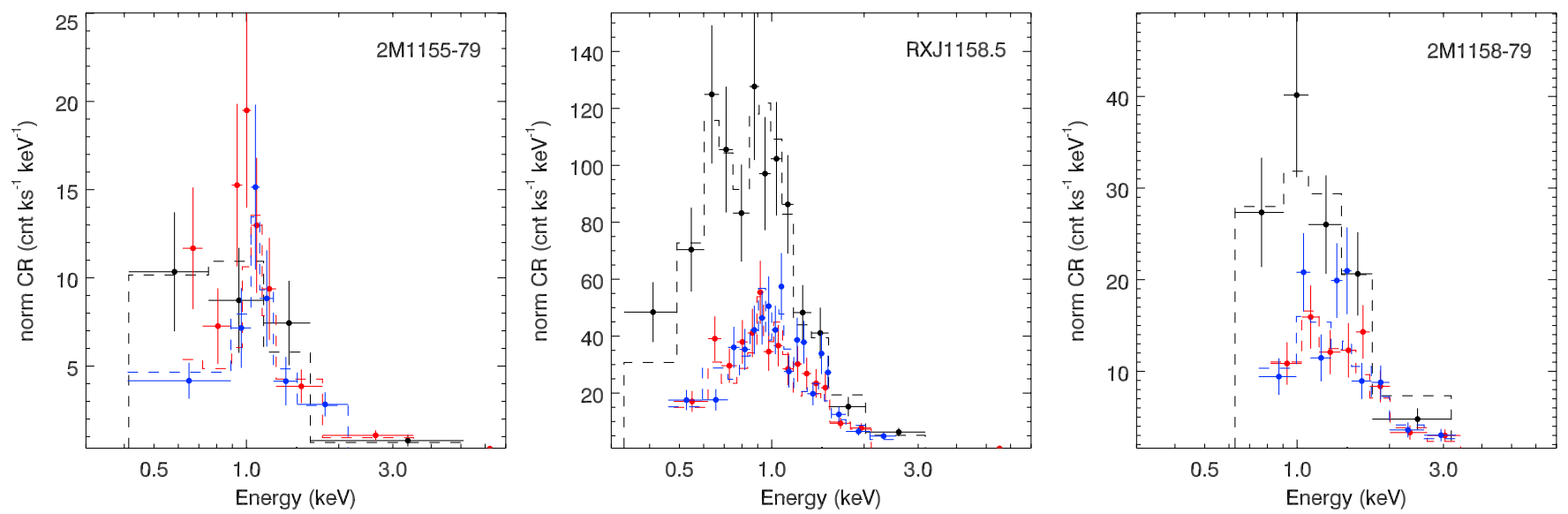

Figure 2. XMM-Newton/EPIC spectra (pn: black points, MOS1: red points, MOS2: blue points) of 2M1155-79 and the two background (Cha cloud) wTTS in the T Cha field (RXJ1158.5 and 2M1158-79) overlaid with best-fit absorbed thermal plasma emission models (histograms, with colors corresponding to instrument data). (A color version of this figure is available in the online journal.) 

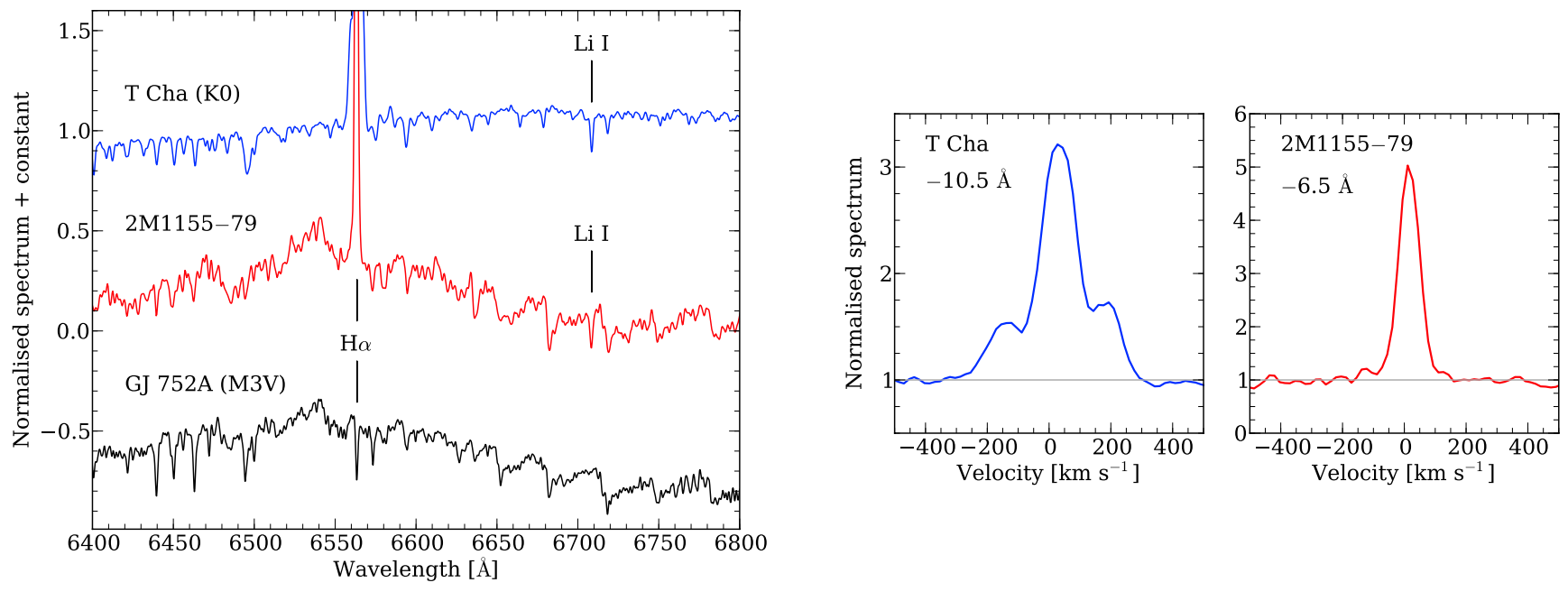

Figure 3. Left: Siding Spring Observatory $2.3 \mathrm{~m}$ telescope 6400-6800 Å spectra of T Cha (blue), 2M1155-79 (red), and M3 field star GJ 752A (black) obtained with the WiFeS spectrograph. The positions of $\mathrm{H} \alpha$ and the $\lambda 6708 \mathrm{Li}$ l line are indicated. Center and right: the $\mathrm{H} \alpha$ emission line regions of the WiFeS spectra of $\mathrm{T}$ Cha and 2M1155-79 (= T Cha B). Line EWs are indicated in each frame.

(A color version of this figure is available in the online journal.)

two-component plasma model to the EPIC spectra of RXJ1158.5 in Table 1, and we display the spectra overlaid with these best-fit models in Figure 2 (a second, lower-temperature component is necessary to model the low-energy regions of the EPIC spectra of RXJ1158.5). The X-ray properties of 2M1155-79 inferred from spectral fitting are similar to those of the background (Cha cloud) wTTS RXJ1158.5 (and are typical of wTTS more generally), consistent with a wTTS classification for 2M1155-79 (Section 3.2). The best-fit model for 2M1158-79 (whose EPIC spectra are evidently somewhat harder than those of 2M1155-79 and RXJ1158.5; Figure 2) indicates a high plasma temperature and very large $L_{X} / L_{\text {bol }}$ ratio, suggesting it may have been undergoing a strong flare during the short exposure targeting $\mathrm{T}$ Cha.

\subsection{Optical Spectroscopy of T Cha and 2M1155-79}

Spectra of T Cha and 2M1155-79 covering the $\mathrm{H} \alpha$ and Li I $\lambda 6708$ spectral region at resolution $\sim 7000$ were obtained with the Australian National University's Siding Spring Observatory (SSO) $2.3 \mathrm{~m}$ telescope and WiFeS spectrometer (Dopita et al. 2007) on 2011 October 12 (Figure 3). Exposure times were $2 \times 600 \mathrm{~s}$ and $300 \mathrm{~s}$ for $2 \mathrm{M} 1155-79$ and T Cha, respectively, and the stars were observed at air mass $\sim 2.5$. Spectra were reduced as described in Riedel et al. (2011); absolute flux calibration was not performed. In the resulting, normalized spectra, both stars display $\mathrm{H} \alpha$ strongly in emission and show strong $\mathrm{Li}$ $\lambda 6708$ absorption lines ( $\mathrm{H} \alpha$ and $\mathrm{Li} \lambda 6708$ line equivalent widths are reported in Table 1). The photospheric absorption features in the spectrum of 2M1155-79 are evidently a close match to those of the M3 field dwarf GJ 752A = HD 180617 (Henry et al. 1994); see Figure 3 (left). Hence, we adopt M3 as the spectral type of 2M1155-79 for purposes of the discussion in Section 3.

\section{DISCUSSION}

\subsection{M1155-79 (= T Cha B): A Likely Wide-separation Companion to TCha}

$\mathrm{Li}$ is rapidly depleted in low-mass pre-MS stars, the $\lambda 6708$ photospheric absorption line becoming difficult to detect in nearby, young mid-M stars (even in high-resolution spectra) by the time such stars are of age $>10$ Myr (Yee \& Jensen 2010). Hence, the detection of $\mathrm{Li}$ absorption in the SSO WiFeS spectrum of 2M1155-79 (Section 2.3), combined with its strong X-ray emission (Section 2.2) and $\mathrm{H} \alpha$ emission (Section 2.2), confirms that this star is young. More specifically, the large $\lambda 6708 \mathrm{Li}$ I EW we measure in the spectrum of 2M1155-79 is comparable to those of mid-M stars in the 8 Myr old TW Hya Association (TWA; Rodriguez et al. 2011) and the $\sim 7$ Myr old $\epsilon$ Cha group (even though 2M1155-79 is among the lowest-mass $\epsilon$ Cha members known; Torres et al. 2008). In contrast, its $\mathrm{Li}$ absorption line $\mathrm{EW}$ is a factor at least 3 larger than those of $M$ stars (of all spectral subtypes) in the $\sim 12$ Myr old $\beta$ Pic Moving Group (Yee \& Jensen 2010). Hence, its Li absorption line strength alone places an upper limit of $\sim 10 \mathrm{Myr}$ on the age of $2 \mathrm{M} 1155-79$.

Adopting the data and methods in Kenyon \& Hartmann (1995), the 2MASS magnitudes and M3 spectral type of 2M1155-79 suggest an effective temperature of $3400 \pm 100 \mathrm{~K}$ (where the error corresponds to an uncertainty of one spectral subclass) and-assuming 2M1155-79, like T Cha, lies at $D=109$ pc (Torres et al. 2008) but, unlike T Cha, suffers negligible reddening (given its low $N_{\mathrm{H}}$; see below)—a bolometric luminosity $\log L_{\text {bol }} / L_{\odot}=-1.16$. The pre-MS evolutionary tracks of Siess et al. (2000), which are appropriate given the age and mass range of interest here (e.g., Lawson \& Feigelson 2001), then indicate an age $\sim 10 \mathrm{Myr}$ and mass just under $\sim 0.3 M_{\odot}$ (Figure 4 ). The former is consistent with the upper limit on the age of 2M1155-79 imposed by its strong $\mathrm{Li}$ absorption. In light of their similar PMs (Section 2.1; see also Terranegra et al. 1999) and the fact that (adopting $D=109 \mathrm{pc}$ ) the two stars fall near the same (age $10 \mathrm{Myr}$ ) theoretical isochrone in Figure 4-an isochrone that is, in turn, very similar to the estimated (7 Myr) age of the $\epsilon$ Cha group with which $\mathrm{T}$ Cha is kinematically associated (Torres et al. 2008, and references therein) - it appears that $\mathrm{T}$ Cha and 2M1155-79 are equidistant, coeval, and comoving. The latter star is therefore most likely a newly identified member of the $\epsilon$ Cha Association.

Furthermore, given the low surface density of known members of the $\epsilon$ Cha group $\left(\sim 0.18 \mathrm{deg}^{-2}\right.$; Torres et al. 2008), the Poisson probability $P$ that T Cha and 2M1155-79 represent a chance alignment of two (otherwise unrelated) $\epsilon$ Cha 


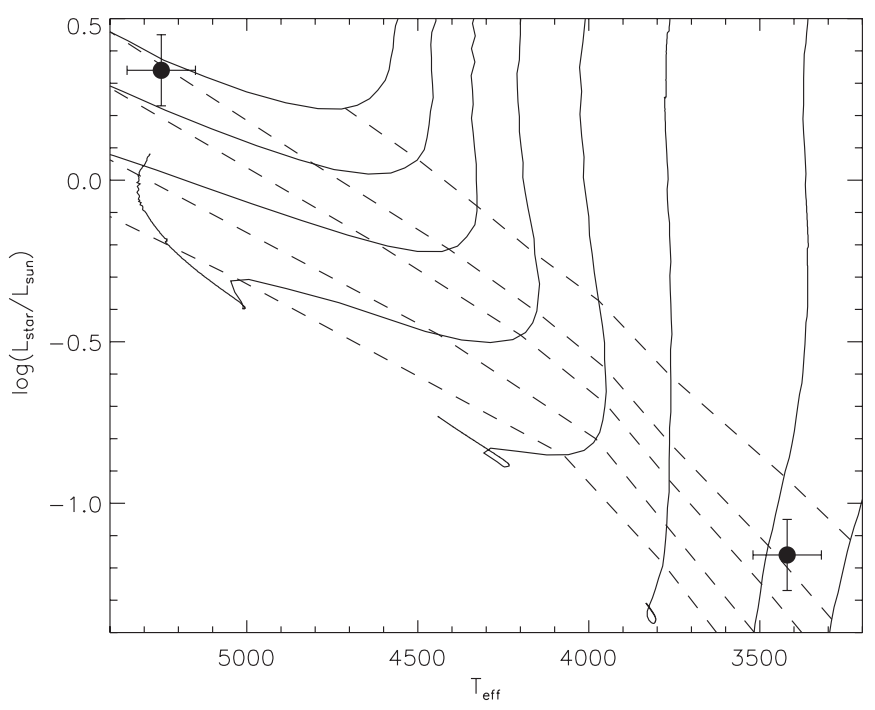

Figure 4. H-R diagram positions of $\mathrm{T}$ Cha $\mathrm{A}$ (point at upper left) and 2M1155-79 (= T Cha B; point at lower right) overlaid on pre-MS tracks from Siess et al. (2000). Error bars reflect estimated uncertainties of $\sim 25 \%$ in luminosity (dominated by distance uncertainties) and $\sim 100 \mathrm{~K}$ in effective temperature. The evolutionary tracks (solid lines) correspond to masses of 0.2 , $0.3,0.5,0.7,0.9,1.1,1.3$, and $1.5 M_{\odot}$ (from lower right to upper left), while the isochrones (dashed lines) correspond to ages of 5, 10, 15, 25, and $40 \mathrm{Myr}$ (from upper right to lower left).

Association stars within the $\left(\sim 0.2 \mathrm{deg}^{2}\right) X M M /$ EPIC field is vanishingly small $\left(P \approx 6 \times 10^{-4}\right)$. We therefore assert that these two stars very likely comprise a very wide (projected separation $\sim 38 \mathrm{kAU}$ ) binary system; hence, hereafter, we refer to $2 \mathrm{M} 1155-79$ as $\mathrm{T}$ Cha B.

\subsection{The Contrasting Natures of T Cha A and B}

The $\mathrm{H} \alpha$ emission line strength of $\mathrm{T}$ Cha displayed in our spectrum (Table 1 and Figure 3, center) lies near the middle of the (wide) range previously measured for this star, and is only marginally consistent with "classical" (as opposed to weaklined) T Tauri star status (the $\mathrm{T} \mathrm{Cha} \mathrm{H} \alpha$ line has also occasionally been observed in absorption; Torres et al. 2008; Schisano et al. 2009). The $\mathrm{H} \alpha$ line width at $10 \%$ of peak intensity at the time of our observations, $W_{10}=475 \mathrm{~km} \mathrm{~s}^{-1}$, would place T Cha among the weakly accreting $\mathrm{T}$ Tauri stars-with an inferred accretion rate of $d M / d t \sim 3 \times 10^{-9} M_{\odot} \mathrm{yr}^{-1}$-given the empirical relationship between $d M / d t$ and $W_{10}$ described in Natta et al. (2004).

In contrast, we measure $W_{10}=149 \mathrm{~km} \mathrm{~s}^{-1}$ for T Cha B (Figure 3, right), suggesting $d M / d t<10^{-11} M_{\odot} \mathrm{yr}^{-1}$ - consistent with the wTTS classification for this star one would determine from its $\mathrm{H} \alpha$ emission-line EW (Table 1; e.g., Barrado y Navascués \& Martín 2003). Furthermore, whereas T Cha A displays a strong mid- to far-infrared excess in 2MASS and Widefield Infrared Survey Explorer ${ }^{10}$ data (e.g., $K-W 3 \sim 2.3$, compared with $K-W 3 \sim 0.5$ for the wTTS RXJ1158.5; Table 1) and is evidently subject to varying degrees of obscuration by its dusty circumstellar disk (Schisano et al. 2009), T Cha B displays only a modest mid-infrared excess $(K-W 3 \sim 0.8)$-although the star evidently has a substantial $\sim 20 \mu \mathrm{m}$ flux excess (as does the background wTTS 2M1158-79; Table 1), suggesting a significant mass of cool circumstellar dust. The presence of disk gas

\footnotetext{
10 The Wide-field Infrared Survey Explorer is a joint project of the University of California, Los Angeles, and the Jet Propulsion Laboratory/California Institute of Technology, and is funded by NASA.
}

around $\mathrm{T} \mathrm{Cha} \mathrm{A}$ - and the apparent absence of such intervening disk gas, along the line of sight to $\mathrm{T}$ Cha $\mathrm{B}$ - is also evident in the large contrast between their respective X-ray absorbing columns (Table 1).

\section{CONCLUSIONS}

T Cha is the third prominent example of a nearby $(D \lesssim$ 100 pc, "old" (age $\sim 10$ Myr) yet still actively accreting, K-type pre-MS star/disk system (the others being TW Hya and V4046 Sgr) that is now known to feature a candidate lowmass companion at very large $(\sim 12-40 \mathrm{kAU})$ separation. The projected separation of the apparent T Cha A/B pair, $\sim 38 \mathrm{kAU}$, is very similar to that of the TWA brown dwarf candidate TWA 28 from TW Hya itself ( $\sim 1 \mathrm{kAU}$; Teixeira et al. 2008), and is much larger than the projected separation of V4046 Sgr AB and C[D] $(\sim 12.4 \mathrm{kAU}$; Kastner et al. 2011). Other examples of nearby, $\sim 10$ Myr old binary systems with components at wide ( 3-13 kAU) separation that feature dusty circumstellar disks are TWA members HR 4796 (TWA 11; Kastner et al. 2008) and TWA 30 (whose two components also display evidence for ongoing accretion; Looper et al. 2010). The projected separations of four of these systems (T Cha A and B; TW Hya and TWA 28; V4046 Sgr AB and C[D]; and HR 4796 AB and $C$ ) rival or exceed those of the widest known main-sequence binaries in the solar neighborhood ( $20 \mathrm{kAU}$; Close et al. 1990). It may be that we are observing these young binary systems at a "fragile" stage of their evolution, during which they are particularly susceptible to dissolution (e.g., via encounters with the older field star population). Indeed, the large separation of $\mathrm{T}$ Cha A and B implies an orbital period 5 Myr, similar to the age of the system itself. High-resolution spectroscopy and precise PM determinations - capable of establishing the relative space motion of the two stars to within $\sim 0.1 \mathrm{~km} \mathrm{~s}^{-1}$ —are required to ascertain whether they are in fact gravitationally bound.

Furthermore, depending on the nature of the substellar companion orbiting within the inner hole of the $\mathrm{T}$ Cha disk (Huélamo et al. 2011), T Cha-like $\beta$ Pic Moving Group member V4046 Sgr and TWA members HD 98800, Hen 3-600, and HR 4796-might be considered a hierarchical binary system with long-lived disk. The presence of wideseparation companions could be pointing to the profound effects of companion-disk interactions, and perhaps hierarchical binary dissolution, on the conditions and timescales for planet formation in such systems (Kastner et al. 2011, and references therein). In particular, given the advanced ages of these five disk-retaining hierarchical binaries, one might speculate that, in each case, the presence of a companion(s) has either inhibited planet formation processes (see also Prato et al. 2001) or extended the time available for such processes well beyond the "nominal" few Myr typically inferred for disk lifetimes (e.g., Currie et al. 2009, and references therein).

We thank the anonymous referee for helpful comments and suggestions. This research was supported by grants to RIT from the National Science Foundation (award AST-1108950) and the NASA Astrophysics Data Analysis Program (award NNX09AC96G).

\section{REFERENCES}

Alcala, J. M., Krautter, J., Schmitt, J. H. M. M., et al. 1995, A\&AS, 114, 109 Andrews, S. M., Czekala, I., Wilner, D. J., et al. 2010, ApJ, 710, 462 Barrado y Navascués, D., \& Martín, E. L. 2003, AJ, 126, 2997 
Close, L. M., Richer, H. B., \& Crabtree, D. R. 1990, AJ, 100, 1968

Currie, T., Lada, C. J., Plavchan, P., et al. 2009, ApJ, 698, 1

Dodson-Robinson, S. E., \& Salyk, C. 2011, ApJ, 738, 131

Dopita, M., Hart, J., McGregor, P., et al. 2007, Ap\&SS, 310, 255

Güdel, M., Lahuis, F., Briggs, K. R., et al. 2010, A\&A, 519, A113

Henry, T. J., Kirkpatrick, J. D., \& Simons, D. A. 1994, AJ, 108, 1437

Huélamo, N., Lacour, S., Tuthill, P., et al. 2011, A\&A, 528, L7

Kastner, J. H., Sacco, G. G., Montez, R., et al. 2011, ApJ, 740, L17

Kastner, J. H., Zuckerman, B., \& Bessell, M. 2008, A\&A, 491, 829

Kastner, J. H., Zuckerman, B., Weintraub, D. A., \& Forveille, T. 1997, Science, 277,67

Kenyon, S. J., \& Hartmann, L. 1995, ApJS, 101, 117

Lawson, W., \& Feigelson, E. D. 2001, in ASP Conf. Ser. 243, From Darkness to Light: Origin and Evolution of Young Stellar Clusters, ed. T. Montmerle \& P. André (San Francisco, CA: ASP), 591

Looper, D. L., Bochanski, J. J., Burgasser, A. J., et al. 2010, AJ, 140, 1486

Mathieu, R. D. 1994, ARA\&A, 32, 465
Natta, A., Testi, L., Muzerolle, J., et al. 2004, A\&A, 424, 603

Prato, L., Ghez, A. M., Piña, R. K., et al. 2001, ApJ, 549, 590

Riedel, A. R., Murphy, S. J., Henry, T. J., et al. 2011, AJ, 142, 104

Rodriguez, D. R., Bessell, M. S., Zuckerman, B., \& Kastner, J. H. 2011, ApJ, 727,62

Schisano, E., Covino, E., Alcalá, J. M., et al. 2009, A\&A, 501, 1013

Siess, L., Dufour, E., \& Forestini, M. 2000, A\&A, 358, 593

Teixeira, R., Ducourant, C., Chauvin, G., et al. 2008, A\&A, 489, 825

Terranegra, L., Morale, F., Spagna, A., Massone, G., \& Lattanzi, M. G. 1999, A\&A, 341, L79

Tohline, J. E. 2002, ARA\&A, 40, 349

Torres, C. A. O., Quast, G. R., Melo, C. H. F., \& Sterzik, M. F. 2008, in Handbook of Star Forming Regions, Vol. II: The Southern Sky, ASP Monograph Publications, Vol. 5, ed. B. Reipurth (San Francisco, CA: ASP), 757

Whittet, D. C. B., Prusti, T., Franco, G. A. P., et al. 1997, A\&A, 327, 1194

Yee, J. C., \& Jensen, E. L. N. 2010, ApJ, 711, 303

Zacharias, N., Finch, C., Girard, T., et al. 2010, AJ, 139, 2184

Zuckerman, B., Rhee, J. H., Song, I., \& Bessell, M. S. 2011, ApJ, 732, 61 